\title{
Insulating, superconducting and large-compressibility phases in nanotube ropes
}

\author{
J. V. Alvarez and J. González \\ Instituto de Estructura de la Materia. Consejo Superior de Investigaciones Científicas. Serrano 123, 28006 Madrid. Spain.
}

(August 13, 2018)

\begin{abstract}
The superconducting properties of carbon nanotube ropes are studied using a new computational framework that incorporates the renormalization of intratube interactions and the effect of intertube Coulomb screening. This method allows to study both the limits of thin and thick ropes ranging from purely one-dimensional physics to the setting of three-dimensional Cooper-pair coherence, providing good estimates of the critical temperature as a function of the rope physical parameters. We discuss the connection of our results with recent experiments.
\end{abstract}

71.10.Pm,74.50.+r,71.20.Tx

Introduction.- Carbon nanotubes offer nowadays a great potential for technological applications. They display semiconducting or metallic properties depending on the helicity of the tubule [1], what is a useful feature to build electronic devices. The electron correlations are significant in the metallic nanotubes $[2-5]$, explaining the experimental observation of features characteristic of Luttinger liquid behavior [6,7]. Quite remarkably, superconducting correlations have been also observed, in the form of the proximity effect $[8,9]$ as well as in the sharp decrease of the resistance at low temperatures [10].

The superconducting transitions reported in Ref. [10] have been measured in various samples of nanotube ropes. These are supposed to be made of a large number $(\sim 300)$ of single-walled nanotubes, and in all cases the critical temperature has been found below $1 \mathrm{~K}$. In a different kind of experiment [11], strong superconducting correlations have been observed in individual nanotubes of very small diameter placed in a zeolite matrix. It has been claimed that such correlations should correspond to a critical temperature of the order of $\sim 15 \mathrm{~K}$ [11].

An explanation of the superconductivity (SC) in the nanotube ropes has been already proposed $[12,13]$. It has been argued that, as long as the Cooper pairs are formed at zero total momentum, they do not find the obstacle that single electrons have to tunnel between neighboring nanotubes due to the misalignement of the lattices $[14,15]$. The existence of a superconducting transition is possible in the ropes as the coherence in the transverse directions is established through the tunneling of Cooper pairs between the nanotubes [13]. In this scenario it has been assumed that the screening of the Coulomb repulsion is the dominant effect in large nanotube ropes and that the backscattering and Umklapp interactions are not essential to explain the SC of these systems.

In this Letter we propose a new computational framework to deal simultaneously with the Coulomb screening due to the intertube interactions and the scaling of all the intratube interactions in the low-energy theory. In this way we will obtain a precise estimate of the critical temperatures that can be reached. The interplay between all the possible interactions in the scaling procedure will allow us to investigate the development of $3 \mathrm{D}$ coherence in the rope, as well as to discover new phases which correspond to different charge instabilities in the system.
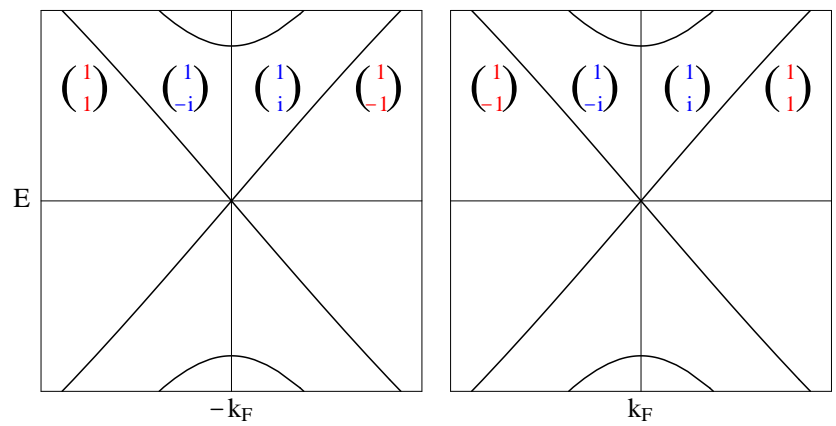

FIG. 1. Plot of the low-energy linear branches of carbon nanotubes. The spinors in the inside (outside) correspond to the relative electron amplitudes in the two sublattices of graphene rolled up to form a zigzag (armchair) nanotube.

Electron-phonon interactions.- The effective interaction coming from the exchange of phonons may lead to attraction or repulsion between the electrons, depending on the particular channel of interaction considered $[16,17]$. We will follow the convention of classifying the four-fermion interactions into different channels with respective coupling constants $g_{i}^{(j)}[18]$. The lower index discerns whether the interacting particles shift from one Fermi point to the other $(i=1)$, remain at different Fermi points $(i=2)$, or they interact near the same Fermi point $(i=4)$. The upper label follows the same rule to classify the different combinations of left-movers and right-movers, including the possibility of having Umklapp processes $(j=3)$.

Let us focus on the opposite cases of armchair and metallic zigzag nanotubes [1]. All of them have two pairs of linear branches in their band structure, crossing at respective points at the Fermi level (for undoped nanotubes) as shown in Fig. 1. Obviating the momentum dependence of the electron modes, the relative amplitudes between points in the two sublattices of the honeycomb 
lattice are given by the spinors in Fig. 1 [19].

Using the fact that the electron modes with opposite momenta have complex conjugate amplitudes, it can be shown that the contributions from phonon exchange to $g_{1}^{(1)}, g_{1}^{(2)}, g_{2}^{(1)}, g_{2}^{(2)}$ and $g_{4}^{(1)}$ are negative, for frequencies below the characteristic phonon energy. However, the contributions from Umklapp processes to $g_{1}^{(3)}, g_{2}^{(3)}$ and $g_{4}^{(3)}$ have the opposite sign, since the respective products of electron-phonon couplings turn out to be negative [17]. We recall that the electron-phonon couplings are given by a sum involving products of electron modes in nearestneighbor sites [16]. Thus, the contributions to $g_{4}^{(2)}$ and $g_{2}^{(4)}$ have attractive character in the zigzag nanotubes, due to the relation of conjugation between the electron modes, but they have repulsive character in the armchair nanotubes, since only one of the two scattered modes reverses its sign there when shifting to a neighboring site.

The signs of the different contributions are based on symmetry rules, and they hold irrespective of whether the exchanged phonons are optical or acoustic. The energy of the latter at momentum $2 k_{F}$ is close to the energy of optical phonons near $k=0$, so that we will assume equal strength in absolute value, $|g|$, for all the four-fermion interactions arising from the exchange of phonons with a typical Debye frequency between 0.1 and $0.2 \mathrm{eV}$.

We will deal with effective interactions coming from the exchange of phonons within the same nanotube. Interactions mediated by the exchange of phonons in different nanotubes require mainly the coupling of out-of-plane phonons, which have a much lower energy scale. These effects have been studied in Ref. [20], with the result that the intratube superconducting order parameter is more relevant than the intertube one, when the system is in the regime with dominant attractive interactions.

Coulomb screening.- - Regarding the Coulomb interaction, it has been shown that the strength of the backscattering and Umklapp processes mediated by the Coulomb potential is reduced by a relative factor $\sim$ $0.1 a / R$, in terms of the ratio of the lattice spacing $a$ to the nanotube radius $R[2-4]$. This means that, for typical nanotubes in a rope, these processes can be safely neglected in favor of the contributions from phonon exchange, whenever the strength in absolute value $|g|$ of the latter is above $\sim 0.05 v_{F}$. Therefore, the competition between the long-range Coulomb interaction and the effective interaction from phonon exchange takes place in the channels with small momentum transfer corresponding to the couplings $g_{2}^{(2)}, g_{2}^{(4)}, g_{4}^{(2)}$ and $g_{4}^{(4)}$.

Dealing for the time being with such small momentumtransfer interactions, the dressed vertices are given by self-consistent equations of the type shown in Fig. 2. It has to be realized that, in the case of a rope, there are interaction vertices $D_{i}^{(j)}$ for currents within the same nanotube as well as interaction vertices $V_{i}^{(j)}$ between cur- rents at different metallic nanotubes. If the number of them is $n$, this is also the number of different terms in the sum that appears in Fig. 2.

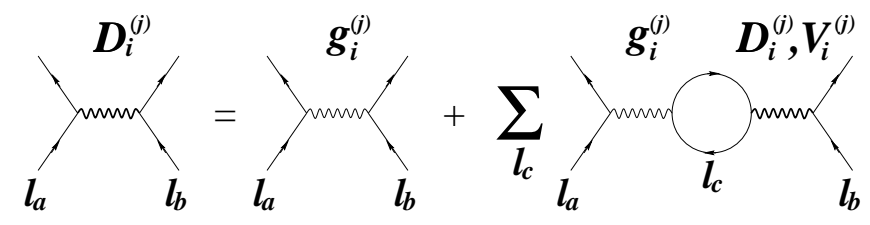

FIG. 2. Diagrams contributing to the screening of Coulomb interactions between currents $l_{a}, l_{b}$ of well-defined chirality and spin inside a rope. The second term takes into account the polarization of the $n$ metallic nanotubes in the rope.

The self-consistent equations for the intratube $D_{i}^{(j)}$ and the intertube $V_{i}^{(j)}$ vertices can be solved in the same fashion as for the simpler case of the Luttinger model [21]. In order to obtain a solution in the form of coupling constants, we have taken the static approximation in the polarization operators. We have also chosen an average strength for the Coulomb potential $v=\left(e^{2} / 2 \pi\right) \log \left|k_{c} / k_{0}\right|$, where $k_{c}$ is the short-distance cutoff and $k_{0} \sim 10^{-3} k_{c}$ accounts for the long-distance average.

The appropriate bare values of the couplings are $g_{4}^{(4)}=$ $v+g, g_{4}^{(2)}=v \mp g, g_{2}^{(2)}=v+g$ and $g_{2}^{(4)}=v \mp g$, where $g<0$ is the contribution from phonon exchange. The upper sign applies to the armchair nanotubes and the lower sign to the zigzag nanotubes. In the former case, the solution of the self-consistent equations is

$$
\begin{aligned}
& D_{4}^{(4)}+D_{4}^{(2)}=D_{2}^{(2)}+D_{2}^{(4)}=\frac{2 v}{1+4 n v / \varepsilon_{0} \pi v_{F}} \\
& D_{4}^{(4)}-D_{4}^{(2)}=D_{2}^{(2)}-D_{2}^{(4)}=\frac{2 g}{1+4 g / \varepsilon_{0} \pi v_{F}}
\end{aligned}
$$

In the case of the zigzag nanotubes, we have

$$
D_{i}^{(j)}=\frac{v+g+4 g(n v+g) / \varepsilon_{0} \pi v_{F}}{\left(1+4 g / \varepsilon_{0} \pi v_{F}\right)\left(1+4(n v+g) / \varepsilon_{0} \pi v_{F}\right)}
$$

for all of the four dressed couplings. The effect of the Coulomb screening is well captured in the expression of the dressed vertices. The RPA-like diagrams considered screen repulsive interactions and enhance attractive interactions, and the latter always prevail for sufficiently large values of $n$. For $4|g| / \varepsilon_{0} \pi v_{F}$ close to 1 , strong nonperturbative effects arise from the same kind of singularity already observed in the bosonization picture $[22,13]$. The limit $n \rightarrow 0, g \rightarrow 0$ recovers the bare vertices used in precedent studies of SC in isolated nanotubes $[17,18]$.

Intratube scaling equations. - The preceding physical description has to be completed by incorporating the effect of the backscattering and Umklapp effective interactions arising from phonon exchange. We set the signs of their respective initial strengths (equal to $|g|$ in 
absolute value) according to the above discussion, and take into account their nontrivial scaling behavior when the frequency probe is progressively reduced. The couplings scale according to anomalous dimensions which can be written in terms of the dressed couplings $D_{i}^{(j)}$. To second order in the backscattering and Umklapp couplings, the scaling equations read

$$
\begin{aligned}
\partial g_{1}^{(2)} / \partial l= & \frac{1}{\pi v_{F}}\left(D_{4}^{(2)}-D_{2}^{(2)}\right) g_{1}^{(2)}+\frac{1}{\pi v_{F}}\left(g_{4}^{(3)} g_{1}^{(3)}\right. \\
& \left.-g_{2}^{(1)} g_{1}^{(1)}\right) \\
\partial g_{1}^{(3)} / \partial l= & \frac{1}{\pi v_{F}}\left(D_{4}^{(2)}+D_{2}^{(2)}\right) g_{1}^{(3)}+\frac{1}{\pi v_{F}}\left(-2 g_{1}^{(3)} g_{1}^{(1)}\right. \\
& \left.+g_{2}^{(3)} g_{1}^{(1)}+g_{4}^{(3)} g_{1}^{(2)}\right) \\
\partial g_{2}^{(1)} / \partial l= & \frac{1}{\pi v_{F}}\left(D_{4}^{(2)}-D_{2}^{(2)}\right) g_{2}^{(1)}+\frac{1}{\pi v_{F}}\left(g_{4}^{(1)} g_{1}^{(2)}\right. \\
& \left.-2 g_{4}^{(1)} g_{2}^{(1)}+g_{4}^{(3)} g_{1}^{(3)}-g_{4}^{(3)} g_{2}^{(3)}-g_{1}^{(2)} g_{1}^{(1)}\right) \\
\partial g_{2}^{(3)} / \partial l= & \frac{1}{\pi v_{F}}\left(D_{4}^{(2)}+D_{2}^{(2)}\right) g_{2}^{(3)}+\frac{1}{\pi v_{F}}\left(g_{4}^{(1)} g_{1}^{(3)}\right. \\
& \left.-2 g_{4}^{(1)} g_{2}^{(3)}+g_{4}^{(3)} g_{1}^{(2)}-g_{4}^{(3)} g_{2}^{(1)}\right) \\
\partial g_{4}^{(3)} / \partial l= & \frac{1}{\pi v_{F}} 2 D_{4}^{(2)} g_{4}^{(3)}+\frac{1}{\pi v_{F}}\left(-g_{4}^{(3)} g_{4}^{(1)}\right. \\
& \left.-2 g_{2}^{(3)} g_{2}^{(1)}+g_{1}^{(3)} g_{2}^{(1)}+g_{2}^{(3)} g_{1}^{(2)}+g_{1}^{(3)} g_{1}^{(2)}\right)
\end{aligned}
$$

where $l$ stands for (minus) the logarithm of the energy scale measured in units of the high-energy cutoff $E_{c}$ (of the order of $\sim 0.1 \mathrm{eV})$. These equations are similar to those obtained in Ref. [18], except for the replacement of $g_{2}^{(2)}, g_{2}^{(4)}, g_{4}^{(4)}$ and $g_{4}^{(2)}$ by the respective dressed couplings. We apply this replacement to the rest of the equations in the model, including those for the small momentum-transfer couplings, which we do not write since they are similar to those in Ref. [18]. In this way we incorporate the finite renormalizations arising from the interaction among the metallic nanotubes in the rope, which set the initial values of the dressed couplings in accordance to Eqs. (1)-(3).

Intertube coherence.- The ropes are made of nanotubes with different helicities and diameters, and this compositional disorder frustrates the formation of any phase with spin-density-wave or charge-density-wave order in the rope. On the other hand, the nanotubes may have strong superconducting correlations. Their strength is given by the combination of the couplings $D_{2}^{(2)}, g_{1}^{(1)}, g_{2}^{(1)}$ and $g_{1}^{(2)}$, which govern the propagation of the Cooper pairs along each nanotube. The interactions corresponding to $g_{1}^{(1)}$ and $g_{2}^{(1)}$ are always attractive, pointing at the enhancement of the superconducting correlations with $s$-wave symmetry at low energies.

In the compositionally disordered ropes, the coherence is established by the tunneling of Cooper pairs between neighboring metallic nanotubes. The amplitude $J$ for this process has a dependence on the energy scale characterized by the anomalous dimension $\Delta$, given by the combination $D_{2}^{(2)}+g_{1}^{(1)}+g_{2}^{(1)}+g_{1}^{(2)}$. The scaling equation for $J$ is [23]

$$
\partial J / \partial l=\Delta J+c t_{T}^{2}
$$

where $t_{T}$ is the intertube hopping for each particle of the Cooper pair, of the order of $\sim 0.01 \mathrm{eV}$. We recall that the tunneling amplitude for single electrons is in general much smaller than this quantity, due to the mismatch between the Fermi points of neighboring nanotubes [14]. This introduces a factor of suppression that sets the single-particle intertube hopping about three orders of magnitude below the estimate for $t_{T}$ [15].

If the generalized backscattering and Umklapp processes are fixed to zero, the system is at the fixed point described in the bosonization picture [13]. That fixed point is in general unstable against chirality-breaking perturbations by which the system flows to a strong-coupling fixed point. Nevertheless, the crossover behavior is very slow, being characterized by a relatively small crossover frequency $\omega_{x o}$. In a large region of the phase diagram the flow is cut off by the onset of the $3 \mathrm{D}$ coherence before $\omega_{x o}$ has been reached. In these conditions, the SC phase remains in the region of influence of the weak-coupling fixed point, which governs then the main physical properties.

Results and discussion.- Following the above approach, we characterize the superconducting transition by looking for a pole $\omega_{c}$ in the $3 \mathrm{D}$ propagator $\mathcal{R}(\omega)$ of the Cooper pairs along the rope. This is related to the SC response function $\mathcal{R}^{(0)}(\omega)$ in the individual nanotubes by the Schwinger-Dyson equation $1 / \mathcal{R}(\omega)=1 / \mathcal{R}^{(0)}(\omega)-\tilde{J}$, where $\tilde{J}$ is the (dimensionless) pair hopping measured in units of the high-energy cutoff.

Moreover, we can study the competition between the $\mathrm{SC}$ and other charge instabilities in the rope by rebosonizing the system at each step in the integration of Eqs. (4)-(8), considering backscattering and Umklapp couplings as small perturbations. The generalized Luttinger liquid parameters of the individual nanotubes take the form

$$
K_{ \pm}=\sqrt{\frac{\pi v_{F}+\left(D_{4}^{(4)} \pm D_{2}^{(4)}\right)-\left(D_{2}^{(2)} \pm D_{4}^{(2)}\right)}{\pi v_{F}+\left(D_{4}^{(4)} \pm D_{2}^{(4)}\right)+\left(D_{2}^{(2)} \pm D_{4}^{(2)}\right)}}
$$

We obtain complementary information about the physical properties of the system by studying the regions where the $K_{ \pm}$parameters either vanish or diverge. This happens when some of the functions given by Eq. (10) develops a branch-cut at some energy scale, what has to be understood as the onset of a phase transition in the system. The different phases which arise in this approach have been represented in Fig. 3, where contour lines of constant critical scale $\omega_{c}$ have been also plotted.

The weak-coupling character of the SC phase allows to estimate the transition temperatures from the values of the pole $\omega_{c}$ shown in Fig. 3. The comparison with the experiments reported in Ref. [10] can be made by recalling 
that the ropes used there have about 100 metallic nanotubes, while the transition temperatures measured are always below $1 \mathrm{~K}$. When compared to the high-energy cutoff, that value corresponds to $l_{c} \approx 7$. From the results shown in Fig. 3, we find that the strength of the attractive interaction $4|g| / \pi v_{F}$ has to be $\approx 0.3$.
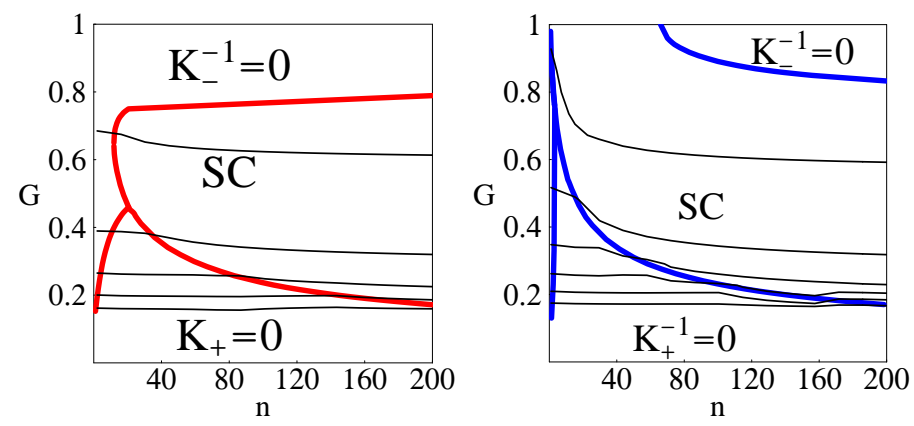

FIG. 3. Phase diagram of undoped armchair (left) and doped zigzag (right) nanotubes as a function of the number of metallic nanotubes $n$ in the rope and the strength of the attractive interaction $G=4|g| / \pi v_{F}$. Thick lines represent the phase boundaries between a 3D phase-coherent superconducting phase (SC) and the $1 \mathrm{D}$ phases characterized by the breaking of the parameters $K_{+}$and $K_{-}$(the narrow phase to the left of the second diagram corresponds to the divergence of the latter). Thin lines are contours of constant critical frequency $\omega_{c}$, starting from above with $\ln \left(E_{c} / \omega_{c}\right)=2,4,6, \ldots$

Given the above estimate of $g$, we conclude that the superconducting correlations have to disappear below a number of metallic nanotubes $n \lesssim 50$, in ropes of the type measured in Ref. [10]. That is, for nanotubes with a typical diameter of $\approx 1.4 \mathrm{~nm}$, the effect of the Coulomb interaction overcomes any source of effective attraction for that range of $n$. This is in agreement with the experimental measures carried out by the collaboration of Ref. [10] in thin ropes with about 40 nanotubes [24].

The lower boundary of the SC phase in Fig. 3 almost coincides with the boundary at which the pair-hopping amplitude ceases to grow large at low energies. When this happens, the nanotubes in the rope start to behave as uncoupled 1D systems, what explains the disappearance of the superconducting transition. A phase with vanishing compressibility appears for low values of $|g|$ in the undoped system. The fact that the regime with $K_{+}=0$ is only present at half-filling makes clear that it corresponds to the extension of the Mott insulating phase found in the studies of carbon nanotubes with respulsive interactions $[4,5]$. In the case of doped nanotubes, the repulsive Umklapp interactions are not present in the system, and the decoupling of the nanotubes in the rope leads instead to a phase with divergent compressibility in the channel of the total charge, as shown in Fig. 3.

Another phase with divergent compressibility arises at strong coupling in the channel of the mismatch of charge in the two gapless subbands. These divergences have the same character of the Wentzel-Bardeen singularity, which is the natural counterpart of the superconducting instability $[22,20]$. A divergent compressibility implies that the density-density correlations become increasingly large, pointing at the onset of a regime where there is no homogeneous descripition of the system. For sufficiently strong attraction, the electronic charge finds then more favorable its macroscopic segregation than the formation of Cooper pairs. We have shown that such a phase may be observable in ropes, either as a consequence of a strong attractive interaction or, in doped systems, from the decoupled behavior of the metallic nanotubes at weak coupling, for temperatures slightly below $1 \mathrm{~K}$.

[1] N. Hamada, S. Sawada and A. Oshiyama, Phys. Rev. Lett. 68, 1579 (1992). R. Saito et al., Appl. Phys. Lett. 60, 2204 (1992).

[2] L. Balents and M. P. A. Fisher, Phys. Rev. B 55, R11973 (1997).

[3] R. Egger and A. O. Gogolin, Phys. Rev. Lett. 79, 5082 (1997); Eur. Phys. J. B 3, 281 (1998).

[4] C. Kane, L. Balents and M. P. A. Fisher, Phys. Rev. Lett. 79, 5086 (1997).

[5] H. Yoshioka and A. A. Odintsov, Phys. Rev. Lett. 82, 374 (1999); Phys. Rev. B 59, R10457 (1999).

[6] M. Bockrath et al., Nature 397, 598 (1999).

[7] Z. Yao et al., Nature 402, 273 (1999).

[8] A. Yu. Kasumov et al., Science 284, 1508 (1999).

[9] A. F. Morpurgo et al., Science 286, 263 (1999).

[10] M. Kociak et al., Phys. Rev. Lett. 86, 2416 (2001).

[11] Z. K. Tang et al., Science 292, 2462 (2001).

[12] J. González, Phys. Rev. Lett. 87, 136401 (2001).

[13] J. González, Phys. Rev. Lett. 88, 076403 (2002); Phys. Rev. B 67, 014528 (2003).

[14] A. A. Maarouf, C. L. Kane and E. J. Mele, Phys. Rev. B 61, 11156 (2000).

[15] H. Stahl et al., Phys. Rev. Lett. 85, 5186 (2000).

[16] R. A. Jishi, M. S. Dresselhaus and G. Dresselhaus, Phys. Rev. B 48, 11385 (1993).

[17] A. Sédéki, L. G. Caron and C. Bourbonnais, Phys. Rev. B 65, 140515 (2002).

[18] Yu. A. Krotov, D.-H. Lee and S. G. Louie, Phys. Rev. Lett. 78, 4245 (1997).

[19] J. González, F. Guinea and M. A. H. Vozmediano, Nucl. Phys. B 406, 771 (1993).

[20] A. De Martino and R. Egger, report cond-mat/0212412.

[21] J. Sólyom, Adv. Phys. 28, 201 (1979).

[22] D. Loss and T. Martin, Phys. Rev. B 50, 12160 (1994).

[23] V. M. Yakovenko, Pis'ma Zh. Eksp. Teor. Fiz. 56, 523 (1992) [JETP Lett. 56, 510 (1992)].

[24] A. Kasumov, private communication. 